The Be Phenomenon in Early-Type Stars, IAU Colloquium 175

ASP Conference Series, Vol. 214, 2000

M. A. Smith, H. F. Henrichs, and J. Fabregat, eds.

\title{
Be Stars in Roche-Lobe Interacting Binaries
}

\author{
A.E. Tarasov \\ Crimean Astrophysical Observatory, Nauchny, Crimea, 98908 Ukraine
}

\begin{abstract}
Different types of massive interacting binaries with Be components are discussed. Due to mass exchange, Be stars in these systems have some peculiar characteristics. Often it is even difficult to find traces of the Be star and/or mass-losing star in the optical region of the spectrum. Using the value of the orbital period as a primary parameter, it is possible to divide all massive interacting binaries into two large groups: short-period or classical Algols and long-period binaries. The last group includes a number of very active mass transfer systems named as W Serpentis. There is also a small group of double systems whose stars do not fill their Roche lobes but have emission lines in the spectrum. Properties of their spectral variability are discussed. Analysis of each group lets us find different behaviours in mass exchange that depend on the size of their Roche lobes.
\end{abstract}

\section{Introduction}

It is well known that most classical interacting binaries have a Be star as one of the components. It is important that subsequent evolution of such systems will generate a variety of double star systems with a Be star primary and compact object companion (black holes, neutron stars or white dwarfs). So, it is clear that we need to obtain enough knowledge about stars undergoing the first stage of mass exchange to understand the evolution of Be stars into other types of double systems.

Because of mass and angular momentum transfer, Be stars are born in interacting binaries as soon as the more massive star of the pair leaves the main sequence. Differential evolution will quickly create a large gap in the H-R Diagram between the two components, even if their masses are very similar. Eventually, the less massive star, still on the main sequence, will have a companion, the erstwhile primary, which evolves to become a late-type giant or supergiant.

Looking at the samples of massive interacting binaries with Be components it is easiest to separate them into two groups, taking into account the value of the orbital period as a primary parameter. The first group contains numerous short period (2-7 days) binaries or "classical Algols". Most of these are dominated by the spectrum of a late B or early A star, have traces of lines from the cool giant and, as a rule, poorly developed and unstable emission in $\mathrm{H} \alpha$. The second, less numerous group contains more massive binaries with a wide range of periods from 6-7 days to a few hundred days. Their spectra contain bright hydrogen emission lines accompanied by the HeI emission or absorption lines and often 


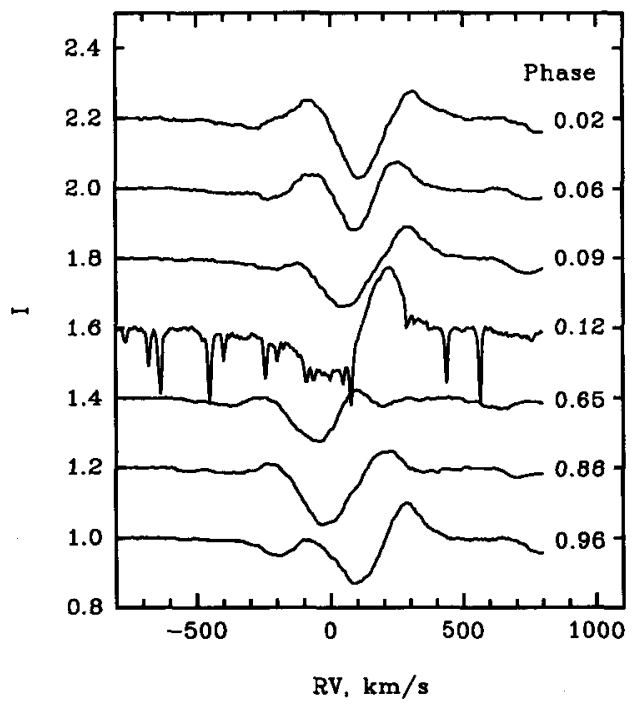

Figure 1. V373 Cas. Variability of $\mathrm{H} \alpha$ profile with the orbital period.

have a rich shell spectrum (Peters 1979). The most active stars Plavec (1980) classifies as W Serpentis binaries, named after one of the best studied system of this type..

\section{Be stars in double systems with both components on the main sequence}

As known from observations, massive binaries with B star components can have a disk around one of them much hotter than a more massive star filling its Roche lobe. It is important to study such systems in detail since they can contribute additional information about the processes of mass and angular momentum transfer before a more massive star leaves the main sequence and fills its Roche lobe. Since this stage of evolution is very short, we know only a few good examples of massive binaries at this stage of evolution.

Let me demonstrate some details of spectral variability of the emission spectrum, physical parameters and evolutionary status of such systems using the example of the recently well studied early-type double system V373 Cas. This double star has an orbital period of $\sim 13.4$ days, moderate orbital eccentricity $(e=0.14)$, and spectral classes of the components B0.5 II and B0.5 III (Lyubimkov et al. 1998). Using broad band $U B V R I$ polarimetry Berdyugin (1998) found an inclination angle of the system of $58.5^{\circ}$ which permitted mass estimates fori the components of $18.6 M_{\odot}$ and $14.2 M_{\odot}$, respectively. The $\mathrm{H} \alpha$ emission has been recently rediscovered by Berdyugin et al. (1995). Some examples of the $\mathrm{H} \alpha$ profiles are shown in Figure 1.

One can see that emission in this double star is associated with the disk around the more massive component. The evolutionary status of the components 


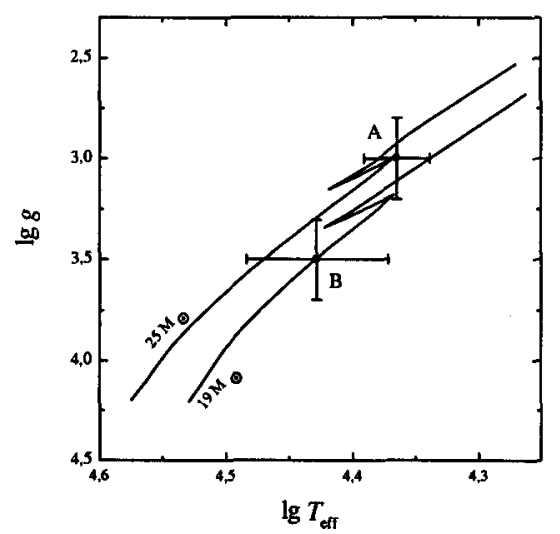

Figure 2. Evolutionary status of the components of V373 Cas.

is shown in Figure 2. In particular, the more massive component is located near the end of or just leaving the main sequence and is still far from filling its Roche lobe.

The evolutionary status of the primary of V373 Cas allows it to be a classical Be star but it is probable that mass loss is caused by the gravitational influence of the secondary star in the eccentric orbit. Additionally, the absence of synchronization or pseudo-synchronization of rotational velocities of the two components at periastron indicates instability of the system. The stars in this system have an excess of rotational velocities, so it is probable that the primary star had not enough time to become synchronized, whereas the secondary is no longer synchronized because mass accretion has already started.

It is not easy to identify double systems at this stage of evolution, but it is possible that the well known and extremely unusual system $\alpha$ Vir (Spica) could be one of the candidates (Lyubimkov et al. 1995; Smith 1985). Unfortunately, it has no noticeable emission in the $\mathrm{H} \alpha$ line.

The other double system that demonstrates similar emission characteristics to V373 Cas is a member of the $\chi$ Per cluster, BD $56^{\circ} 578$. This is an early type double system with a possible orbital period of $\sim 5.2$ days (Krzesiński \& Pigulski 1997). It has faint emission in the $\mathrm{H} \alpha$ line. I present in Figure 3 two examples of $\mathrm{H} \alpha$ profiles with obvious variability of its emission with orbital phase. Fabregat et al. (1996) determined the physical characteristics of the star and found that it is possible to conclude that neither component of the pair reaches the Terminal Age Main Sequence (TAMS). Nevertheless, BD $56^{\circ} 578$ is still too poorly studied to make any final conclusion on its evolutional status.

\section{Be stars in short-period Algols}

Algol-type binary stars are interacting systems in which the cooler G-K III-IV secondary star is transferring mass to its B-A main sequence companion through Roche lobe overflow. These are mostly binaries in a slow phase of mass transfer with rates $\dot{\mathrm{M}} \sim 10^{-11}-10^{-7} M_{\odot} \mathrm{y}^{-1}$. The radius of the primary in this group 


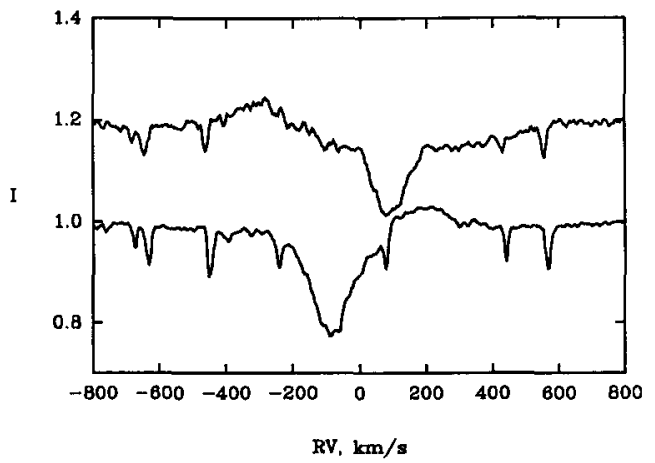

Figure 3. $\mathrm{BD} 56^{\circ} 578$. Example of the orbital variability of the $\mathrm{H} \alpha$ profile.

is a substantial fraction of the binary separation, so the gas stream from the inner Lagrangian point makes a direct impact with the slowly rotating primary. The high-velocity $\left(\sim 500 \mathrm{~km} \mathrm{~s}^{-1}\right)$ gas stream is shocked by the impact with the star, but it partially survives and has enough angular momentum to circle the star and form a variable, asymmetric, disk-like structure which has been called a "transient accretion disk". The resulting circumstellar material consists of the shock region and a second region on or around the mass gainer, in which shock energy is dissipated (Peters 1989).

It is difficult to detect any form of circumstellar material in the spectra of short-period Algols because of the transient nature of the gas, and because emission can only be detected in the observed spectrum during primary eclipse (Kaitchuck \& Honeycutt 1982; Kaitchuck, Honeycutt, \& Schlegel 1985).

Recent spectroscopic study of well known systems such as Algol itself (Richards 1993), TX UMa (Albright \& Richards 1993) and U Sge (Albright \& Richards 1995) show the presence of double-peaked $\mathrm{H} \alpha$ emission features at all phases outside of primary eclipse. These authors performed "full-orbit" $\mathrm{H} \alpha$ spectroscopic investigations. Emission from the circumstellar material was enhanced by subtracting a composite theoretical photospheric $\mathrm{H} \alpha$ profile from the observed profile at each phase. The difference profiles revealed evidence of an asymmetric transient accretion disk and localized high-density $\mathrm{H}$ II regions. Richards, Albright, \& Bowles (1995) used Doppler tomography of the $\mathrm{H} \alpha$ difference profiles of four short-period Algol-type binaries: U CrB, USge, RS Vul and $\beta$ Per, and revealed gas streams due to Roche lobe overflow and disk-like structures around the mass gainer.

Hydrodynamic two-dimensional simulations of the mass transfer of Algol have been conducted by Blondin, Richards, \& Malinowski (1995). They found that the region of highest thermal pressure corresponds to the region of interaction between the gas stream from the secondary and the gas which had circled the primary star. This interaction region near the surface of the primary star and on the line of centre of mass of the two components may be the source of the localized $\mathrm{H} \alpha$ emission. 


\section{Be stars in $W$ Serpentis type binaries}

As soon as the value of the orbital period exceeds $6-7$ days, the gainer becomes small relative to the binary separation and mass transfer from the loser can form a classical accretion disk around the hot companion; now its spectrum can have bright emission lines with similar profiles to those of classical Be stars. According to Plavec (1980), some members of this group are in a short phase of rapid mass exchange and have been named after the prototype as W Serpentis systems.

Most stars of this type have similar luminosities for the loser, gainer, and envelope surrounding the hot star and whole system. Often it is not easy to find traces of their stellar lines in the optical region of the spectrum. Therefore, even finding principal parameters of the orbit is not an easy task. It is only recently that lines of the hottest components in such well known interacting binaries as $\beta$ Lyr (Skulsky 1992; Harmanec et al. 1996) or lines of cool loser in KX And (Floquet et al. 1995; Tarasov, Berdyugina, \& Berdyugin 1998) were found. Similar problems appear in the analysis of light curves. They are strongly distorted by the circumstellar disk and gas streams, preventing orbital solutions for eclipsing binaries from being easily determined. In the case of noneclipsing binaries, the light curves have complex structures due to partial eclipsing of differential parts of the envelope.

Dense gas streams, optically thick disks, and strong mass loss of whole systems form the rich emissien and shell spectrum in the optical and UV regions. W Serpentis systems have bright emission lines of hydrogen, helium and sharp absorption of ions. In the UV part of the spectrum emission lines dominate. Depending on the activity of the systems, they can have mass loss rate $\dot{M}$ from $10^{-7}$ up to $10^{-4} M_{\odot} \mathrm{y}^{-1}$ (Plavec 1980).

Figure 4 and Figure 5 show a few examples of spectra of W Serpentis type stars in the $\mathrm{H} \alpha$ and $\mathrm{HeI} 6678$ regions. All of these stars exhibit very strong double component emission in the $\mathrm{H} \alpha$ line. In most cases, the $\mathrm{R}$ emission peak is brighter than the $\mathrm{V}$ with shifting of the absorption component in the blue part by a few dozen $\mathrm{km} \mathrm{s}^{-1}$. For most of the stars, the $\mathrm{H} \alpha$ line is not strongly variable with the orbital period, and its $\mathrm{P}$ Cyg shape indicates a large value of mass loss from the whole system. For some stars, mass loss is so strong that a rare nebula forms around the system, seen as narrow complex emission features in hydrogen and even forbidden lines. RY Sct is an excellent example of such a system (de Martino et al. 1992; Gehrz et al. 1995).

The shape and variability of the $\mathrm{H} \alpha$ line contain a lot of information about the gas outflow structure of the outer regions of the system, but, as a rule, very little (if any) information about the inner regions around the hot component. It is a different situation with the HeI 6678 line. As seen from our examples, there are great differences in the intensity and shape of this line. In most cases the HeI lines are strongly variable at the orbital period and appear in the Roche lobe of the gainer, even if they are seen in absorption. If the loser star is a cool giant or supergiant star, and the period is long enough, as in the cases of AX Mon and KX And, one can observe the photospheric lines of the loser. Let me demonstrate some difficulties in the interpretation of W Serpentis type systems with a few examples. 


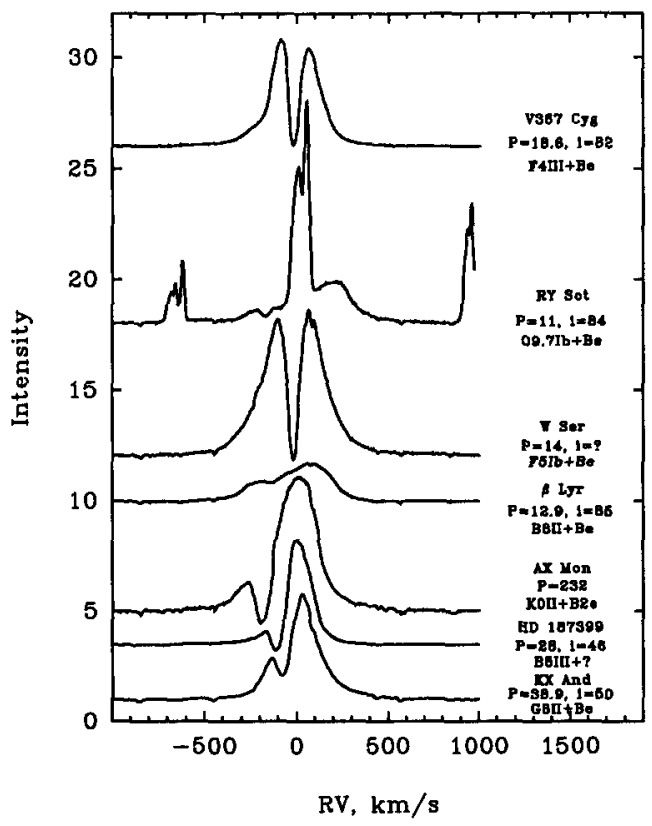

Figure 4. The $\mathrm{H} \alpha$ profiles of some W Serpentis type stars.

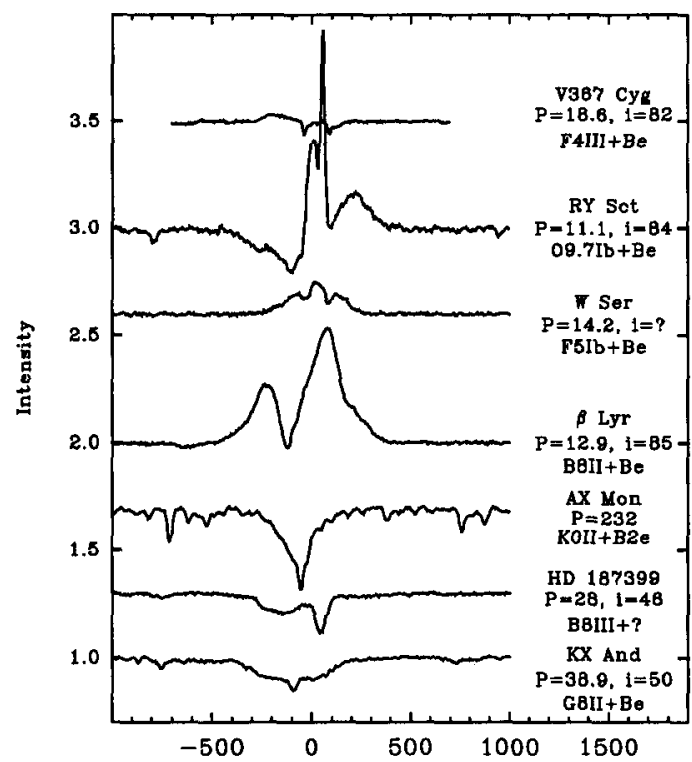

$\mathrm{RV}, \mathrm{km} / \mathrm{s}$

Figure 5. The HeI 6678 profiles of some W Serpentis type stars. 


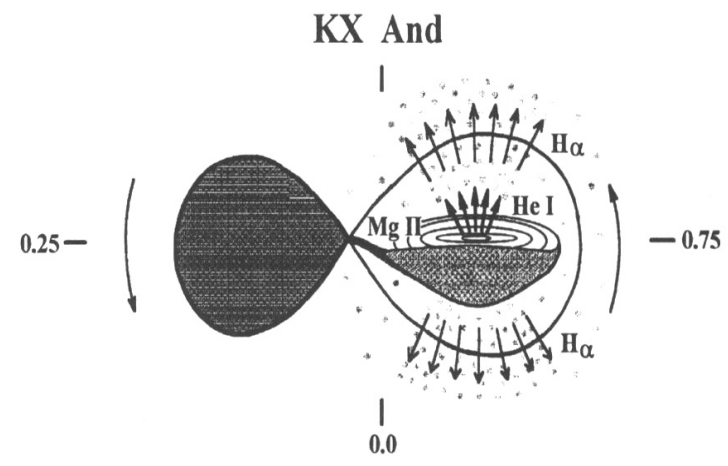

Figure 6. Schematic model of gas streams in KX And. Mg II 4471 and other shell lines appear in the cool disk around the unseen gainer. HeI absorption lines appear above the disk in the hot region near the hot star. $\mathrm{H} \alpha$ and other hydrogen emission lines appear in the gas flows outside the system.

\subsection{KX And}

$\mathrm{KX}$ And is a non-eclipsing system with a cool loser and Be gainer. It has an orbital period of 38.9 days and bright emission in the hydrogen lines. Stefl et al. (1990) failed to find any photospheric lines in its spectrum but separate two groups of sharp absorption metallic shell and broad HeI lines. Both of them form in the Roche lobe of the hot Be component and their orbital radial velocity curves have similar shape and amplitude. The HeI lines look very similar to the photospheric but their $\gamma$ velocities are shifted by about $-100 \mathrm{~km} \mathrm{~s}^{-1}$, suggesting that they form in expanding parts of the envelope. However, there are no traces of the emission P Cyg features in the red wing of the absorption lines that are typical for expanding envelopes.

Later, using CCD observations, Floquet et al. (1995) and Tarasov, Berdyugina \& Berdyugin (1998) found lines from the G8 II loser and demonstrated that the orbit of the system is circular. Using broad band polarimetry, Berdyugin, Berdyugina \& Tarasov (1998) estimated the inclination angle of the system to be $50^{\circ}$ and found independent evidence of gas stream structure in the system. Assuming that radial velocities of absorption lines formed in the disk around the Be component truly reflect the orbital motion of the hot star, Tarasov, Berdyugina, \& Berdyugin (2000) estimate masses of the cool loser as $8 M_{\odot}$ and the hot Be star as $14 M_{\odot}$. Taking into account spectroscopic, polarimetric, and photometric variability of the system, these authors proposed a model of gaseous structures for KX And in which the main fraction of mass loss in the system originates through the polar regions of the disk surrounding the Be component. In Figure 6, a schematic description of gas flows in the system is presented. In this ad hoc model the gas stream leaves the gainer through the inner Lagrangian point and forms an accretion disk around the unseen early type star. Since the Be component has an almost critical rotation velocity, gas can not accrete, so it leaves the system through the polar regions of the gainer. The narrow shell lines in this model are formed in the cool disk and have $\gamma$ velocities the same as that 
for the whole system. The HeI lines form in the hot polar regions and, since the system has a moderate inclination angle, $50^{\circ}$, the emission part of their P Cyg structure is hidden by the optically thick disk. Mass exchange in this situation should be almost completely nonconservative.

AX Mon is a system with similar characteristics to KX And. Since its orbital period is about 232 days, lines of the cool star are easy to find in the spectrum. The HeI lines are in absorption and are shifted by $-70 \mathrm{~km} \mathrm{~s}^{-1}$. The $\mathrm{H} \alpha$ line is not strongly variable and has a similar P Cyg structure to KX And.

\section{2. $\beta$ Lyr}

The typical and best studied example of W Serpentis type stars with a hot gainer is $\beta \mathrm{Lyr}$, an eclipsing binary with an orbital period of 12.9 days. Skulsky (1992) found lines of Si II $6347 \& 6371 \AA$ formed in the photosphere (or disk, Harmanec et al. 1996) around the hidden gainer and estimated the masses of components as $12.5 M_{\odot}$ for the loser and $3 M_{\odot}$ for the gainer. Later, Harmanec et al. (1996) found interferometric and spectroscopic evidence for the existence of an extended jet of gas orientated perpendicularly to the orbital plane of the binary. Using spectropolarimetric observations, Hoffman, Nordsieck \& Fox (1998) confirmed the presence of gas streams located in polar regions of the gainer. According to these investigators, the model of gas streams in $\beta$ Lyr is very similar to the model of KX And described above.

\subsection{HD 187399}

HD 187399 is another example of a massive interacting binary with a hot giant loser B8III, orbital period of 28 days, and a large value of orbital eccentricity $e=0.39$ (Hutchings \& Redman 1973). This non-eclipsing double system has complex orbital variability (Pavlovski et al. 1979). Recently, using UBVRI polarimetry, Berduygin, \& Tarasov (1998) estimated the inclination angle of the system as $46.4^{\circ}$. The system has bright and slightly variable emission in $\mathrm{H} \alpha$ with the shape of the line profile similar to KX And and AX Mon. I present in Figure 7 spectra obtained in the region of the HeI 6678 line. As is seen from the figure, the line profiles have complex structure: the component from the hot loser moves back and forth across a relatively stable absorption structure shifted by about $-100 \mathrm{~km} \mathrm{~s}^{-1}$. The Be star in this system is completely hidden; none of its photospheric lines have been found. As in the cases of $\mathrm{KX}$ And and $\beta \mathrm{Lyr}$, the presence of a shifted absorption component in the HeI lines can originate in the polar gas streams.

\subsection{BY Cru}

BY Cru is another example of W Serpentis type stars recently studied (Deams, Waelkens, \& Mayor 1997) and Deams \& Waelkens (1997).). It has an orbital period of 106.4 days, an unseen gainer, and a type B8/9 Ia/b loser. It is not an eclipsing binary since its orbital inclination is between $64^{\circ}$ and $76^{\circ}$. The estimated masses of the components are 1.7-1.9 $M_{\odot}$ for the loser and 9-11 $M_{\odot}$ for the gainer. As for other stars of this type, the $\mathrm{H} \alpha$ line shows no variability and its double peaked profile implies that it is formed in a circum-binary envelope. This star has complex variability of the HeI 5876 line profile with a transition from complex quasi-P Cyg emission to absorption. The blue wing shifts up to 


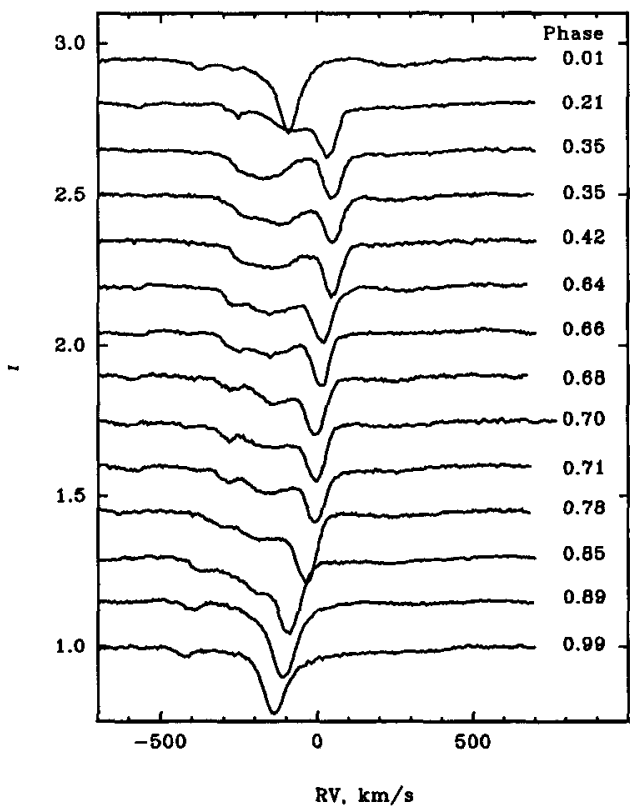

Figure 7. HD 187399. Variability of the HeI 6678 line with the orbital period.

$-500 \mathrm{~km} \mathrm{~s}^{-1}$ at some orbital phases. Unfortunately, the observed profiles do not cover the whole orbital period. but their behaviour appears to lie somewhere between the behaviours of the HeI line profile variability in $\beta$ Lyr and HD 187399 .

\subsection{V367 Cyg}

V367 Cyg shows typical problems that can occur in our study of W Serpentis type double systems. This eclipsing binary system has an orbital period of 18.6 days. Its spectrum exhibits only one photospheric line ( $\mathrm{Mg} \mathrm{II} \mathrm{4481)} \mathrm{and} \mathrm{is} \mathrm{oth-}$ erwise dominated by many shell lines which probably are formed in a common envelope surrounding the whole system. Using $U B V R I$ polarimetry, Berdyugin \& Tarasov (1997) found the inclination angle of this double to be $82.1^{\circ}$. Spectroscopic investigations of the star in the visible region of the spectrum have been made by Tarasov \& Berdyugin (1998). They found a faint broad emission component in the HeI6678 line (see Figure 8).

It apparently forms in the accretion disk around the invisible secondary and shows strong variability of the profile during the orbital period. Analysis of radial velocities of the wings of this line permits estimate in the masses of the binary components of $22 M_{\odot}$ and $11 M_{\odot}$ for the loser and gainer, respectively. This means that the loser still has a larger mass than the gainer. But, their estimations of masses are in strong contradiction with results obtained from photometry by Pavlovski, Schneider \& Acan (1992), who give $2.2 M_{\odot}$ and $4.4 M_{\odot}$ for loser and gainer, respectively. Such a large disagreement means that our methods of estimating masses require additional testing. 


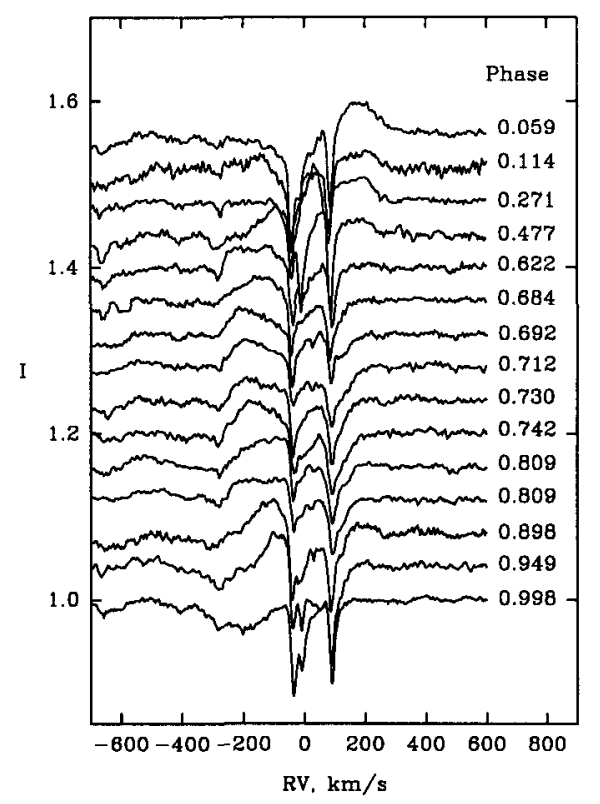

Figure 8. V367 Cyg. Variability of the HeI 6678 line with the orbital period. The numerous absorption lines seen in the spectrum are forming in the common envelope around of the system.

\section{Conclusions}

The fact that practically all massive interacting binaries undergoing the first stage of mass exchange have a $\mathrm{Be}$ component means that some fraction of $\mathrm{Be}$ stars are born in double systems. The main, well known problem is that the mass of the gainer in most cases is many times larger then the mass of loser. Then, if the value of the orbital period is long, demonstration of the binary nature of the system can be a problem. A consequence of this fact is an extremely low quantity of known double Be stars with an evolved low mass secondary. Until now only two such examples have been discovered. By comparison, the quantity of massive X-ray binaries discovered has quickly grown, since they can be identified from ther X-ray characteristics.

One of the ways to estimate the fraction of double systems with Be components are spectroscopic and photometric monitoring of young open clusters rich in Be stars. As the size of telescopes increases, I am sure this task can be achieved soon.

Acknowledgments. I am grateful to C. Brocksopp and Dr. M. Smith for help with corrections to the text. 


\section{References}

Albright, G.E., Richards, M.T. 1993, ApJ, 414, 830

Albright, G.E., Richards, M.T. 1995, ApJ, 441, 806

Blondin, J.M., Richards, M.T., Malinowski, M.L. 1995, ApJ, 445, 939

Berdyugin, A.V. 1998, Pisma Astr. Zh., 24, 84

Berdyugin, A.V., Tarasov, A.E. 1997, AZh, 74, 230

Berdyugin, A.V., Tarasov, A.E. 1998, Pisma Astron Zh., 24, 139

Berdyugin, A.V., Berdyugina, S.V., Tarasov, A.E. 1998, Pisma Astr. Zh., 24, 367

Berdyugin, A.V., Rachkovskaya, T.M., Rostopchin, S.I., Tarasov, A.E. 1995, IBVS, 4158

Daems, K., Waelkens, C., Mayor, M. 1997, A\&A, 317, 823

Daems, K., Waelkens, C. 1997, A\&A, 327, 640

Fabregat, J., Torrejón, J.M., Reig, P. et al. 1996, A\&AS, 119, 271

Floquet, M., Hubert, A.M., Hubert, H. et al. 1995, A\&A, 294, 227

Gehrz, R.D., Hayward, T.I., Houck, J.R. et al. 1995, ApJ, 439, 417

Harmanec, P., Morand, F., Bonneau, D. et al. 1996, A\&A, 312, 879

Hoffman, J.L., Nordsieck, K.H., Fox, G.K. 1998, AJ, 115, 1576

Hutchings, J.B., Redman, R.O. 1973, MNRAS, 163, 209

Kaitchuck, R.H., Honeycutt, R.K. 1982, ApJ, 258, 224

Kaitchuck, R.H., Honeycutt, R.H., Schelegel, E.M. 1985, PASP, 97, 1178

Koubský, P., Harmanec, P., Kubát, J. et al. 1997, A\&A, 328, 551

Krzesiński, J., Pigulski, A. 1997, A\&A, 325, 987

Lyubimkov, L.S., Rachkovskaya, T.M., Rostopchin, S.I., Tarasov, A.E. 1995, AZh, 72, 212

Lyubimkov, L.S., Rachkovskaya, T.M., Rostopchin, S.I., Tarasov, A.E.: 1998, AZh, 42, 212

Martino de, D., Vittone, A.A., Rossi, C., Giovannelli, F. 1992, A\&A, 254, 266

Pavlovski, K., Harmanec, P., Horn, J. et al. 1979, IBVS, 1689

Pavlovski, K., Schneider, H., Akan, M.C. 1992, A\&A, 258, 329

Peters, G.J. 1979, in Plavec, M.J., Popper, D.M. and Ulrich, R.K.(eds), Close Binary Stars: Observations and Interpretation, Dordrecht: Reidel, 287

Peters, G.J. 1989, Space Sci. Rev., 50, 9

Plavec, M.J. 1979, in Plavec, M.J., Popper, D.M. and Ulrich, R.K.(eds), Close Binary Stars: Observations and Interpretation, Dordrecht: Reidel, 251

Richards, M.T. 1993, ApJS, 86, 255

Richards, M.T., Albright, G.E., Bowles, L.M. 1995, ApJ, 438, L103

Skulsky, M.Yu. 1992, Soviet Astron. Lett., 18, 287

Smith, M.A. 1985, ApJ, 297, 206

Štefl, S., Harmanec, P., Horn, J. et al. 1990, Bull. Astron. Inst. Czech., 41, 29.

Tarasov, A.E., Berdyugin, A.V. 1998, AZh, 75, 559

Tarasov, A.E., Berdyugina, S.V., Berdyugin A.V. 1998, Pisma Astr. Zh., 24, 316 
Tarasov, A.E., Berdyugin, A.V., Berdyugina S.V. 2000, Pisma Astr. Zh., in press

\section{Discussion}

G. Peters: (comment) Actually, periods for the so-called "classical Algols" can be much longer than the 6 days that you mentioned. There are many in the "classical Algol" category that have periods in the range 6-20 days or more.

A. Tarasov: Most Algols with an orbital period less than 6-7 days have no stable circumstellar disks around the gainer, and that was the main reason I separated them from the others. There are many massive double stars with longer periods that have roughly similar properties to the short period systems which have more stable disk around the gainer. So, the choice of the critical period would depend from the point of view on proceses of mass exchange in the double systems.

G. Peters: What would you expect to observe if one has the scenario that the thick gas stream (or infalling material) strikes the photosphere of the gainer at a shallow angle or tangentially? Would you not then see a "typical" Be star such as CX Dra, AX Mon or TT Hya?

A. Tarasov: Yes, I think that the spectrum of such a system would look almost the same as the "typical" Be star. But I believe that in the case of an interacting binary we can always find some peculiarities like an unusual shell spectrum and orbital variability of the shape of the emission lines. One of the problems is that it is often not easy to separate interacting binaries from Be stars with the evolved low mass secondaries.

G. Peters: If we could observe V367 Cyg (or other W Serpentis system) poleon, what would you expect to see?

A. Tarasov: That is a good question. We would almost certainly detect some hidden W Serpentis type systems seen pole-on without radial velocity variability and with unknown orbital period. In the spectrum of such a star I would expect to see a strong $\mathrm{P}$ Cyg type profile of $\mathrm{H} \alpha$ with a shape as seen for KX And or AXMon. The HeI lines will be seen in absorption with large shifting of their radial velocities on the value about -50 to $-100 \mathrm{~km} \mathrm{~s}^{-1}$. I do not exclude the possibility that some emission in the red wing would be seen too. And importantly, shell lines appearing in the accretion disk would have normal radial velocities. Polarimetric observation in this case should demonstrate rotation of the position angle with the orbital period.

T. Rivinius: Some Be stars, such as 4 Her (Koubský et al. 1997), do show small absorptions superimposed on $\mathrm{H} \alpha$ emission. In 4 Her this absorption is even periodic, with $\mathrm{P} \sim 46$ days. Could this be a limit to a mass flow in a possible binary?

A. Tarasov: Some double Be non-interacting binaries have absorption or emission features which change over an orbital period. It seems that they appear in the axisymmetrical envelope due to gravitational and/or light interaction. In the classical interacting binaries these features can also be connected with the real mass flow phenomena. But it is not possible to say that it is a limit on the mass exchange because their intensity also depends on the value of the orbital period, spectral types, and evolutionary status of the components. 